\title{
FATORES DE RISCO ASSOCIADOS AO INFARTO AGUDO DO MIOCÁRDIO
}

\section{RISK FACTORS ASSOCIATED WITH ACUTE MYOCARDIAL INFARCTION}

\author{
Maria Stefânya Pereira da Silva ${ }^{1}$ \\ Dara Isabel Vieira de Brito ${ }^{2}$ \\ Pierri Emanoel de Abreu Oliveira ${ }^{3}$ \\ Geane Silva Oliveira ${ }^{4}$ \\ Maria Iranilda Silva Magalhães ${ }^{5}$ \\ Maria Alciene Saraiva de Souza ${ }^{6}$
}

RESUMO: OBJETIVO: Descrever os fatores de riscos associados ao infarto agudo do miocárdio. MÉTODO: Trata-se de uma revisão integrativa da literatura, com base na pergunta norteadora: quais os fatores de riscos associados ao Infarto Agudo do Miocárdio (IAM)? Em sequência foram realizadas uma seleção de artigos publicados nas bases de dados SCIELO que foram encontrados 2.383 artigos e LILACS, onde foram descobertos 3.873 artigos, na qual as buscas dessas bases de dados ocorreram no período de fevereiro a dezembro de 2017. Utilizando os seguintes descritores: "infarto", AND "sinais e sintomas", AND "fatores de riscos". Foram usados como critérios de inclusão: artigos publicados em português, texto completo, disponível e que alcançaram os objetivos propostos, indexados nos referidos bancos de dados nos últimos cinco anos. RESULTADOS: Dos dez artigos selecionados, $20 \%$ (02) são do ano de 2012, 20\% (02) são do ano de 2014, $10 \%$ (01) do ano de 2015, 40\% (04) são do ano de 2016 e 10\% (01) são do ano de 2017. Com base na análise dos artigos utilizados na revisão integrativa, foi verificado que os fatores de riscos associados ao IAM são: HAS, DM, Tabagismo, Sedentarismo, Obesidade, Consumo de Álcool Excessivo. CONCLUSÃO: Este estudo mostra os principais fatores que desencadeiam o IAM evidenciando o grau elevado de morbimortalidade, mostrando que a população precisa ter a consciência de hábitos saudáveis, tendo também o conhecimento sobre todos os fatores para que haja uma prevenção precoce, evitando assim, essa enfermidade.

PALAVRAS CHAVE: Fatores de Riscos. Infarto. Sinais e Sintomas.

\footnotetext{
${ }^{1}$ Graduanda do curso em Biomedicina pela Faculdade Santa Maria, Cajazeiras-PB.

2 Biomédica, Mestre, Docente da Faculdade Santa Maria - FSM, Cajazeiras - PB.

${ }^{3}$ Biomédico, Especialista, Docente da Faculdade Santa Maria - FSM, Cajazeiras - PB.

4 Enfermeira, Mestre, Docente da Faculdade Santa Maria - FSM, Cajazeiras - PB.

${ }^{5}$ Biomédica, Mestre, Docente da Faculdade Santa Maria - FSM, Cajazeiras - PB.

${ }^{6}$ Biomédica, Mestre, Docente da Faculdade Santa Maria - FSM, Cajazeiras - PB.
} 
ABSTRACT: OBJECTIVE: To describe the risk factors associated with acute myocardial infarction. METHODOLOGY: It is an integrative review of the literature, based on the guiding question: what are the risk factors associated with AMI? In the sequence, a selection of articles published in the SCIELO databases was carried out, which found 2,383 articles and LILACS, where 3,873 articles were discovered, in which searches of these databases took place in the period from February to December 2017. Using the following descriptors: "Infarction", AND "signs and symptoms", AND "risk factors". Inclusion criteria were: articles published in Portuguese, full text, available and that will reach the proposed objectives indexed in said databases in the last five years. RESULTS: Of the 10 articles selected, $20 \%$ (02) are from the year 2012, 20\% (02) are from the year 2014, 10\% (01) from the year 2015, 40\% (04) (01) are from the year 2017. Based on the analysis of the articles used in the integrative review, it was verified that the risk factors associated with $A M I$ are: SAH, MD, Smoking, Sedentary lifestyle, Obesity, Excessive Alcohol Consumption. CONCLUSION: This study shows the main factors that trigger AMI evidencing the high degree of morbidity and mortality, showing that the population needs to be aware of healthy habits, and also knowledge about all factors for early prevention, thus avoiding this disease.

KEYWORDS: Risk Factors. Infarction. Signs and Symptoms. 


\section{INTRODUÇÃO}

O Infarto Agudo do Miocárdio (IAM) é uma doença cardiovascular grave, ou seja, é uma Síndrome Isquêmica Miocárdica Instável (SIMI) e sua principal causa é o acúmulo de placa aterosclerótica, ocasionando oclusão na parede do vaso, dificultando a passagem do sangue para órgãos, células e tecidos. É um enorme desafio para os profissionais de saúde detectar precocemente a placa antes dos sintomas clínicos (MERTINS et al., 2016).

Nos últimos anos, estudos epidemiológicos apresentam um crescimento de doenças cardiovasculares, dos quais o IAM está em destaque devido a sua maior magnitude e severidade. Em 2011, cerca de 20 milhões de pessoas apresentaram doenças do aparelho cardiovascular em todo o mundo, dos quais cerca de 12 milhões foram vitimas fatais do IAM. Sendo que, as doenças cardiovasculares consistem na principal causa de morte no Brasil, tendo sido responsável por cerca de 29\% dos óbitos em 2009 (HUGUENIN et al., 2016).

Existem vários fatores de riscos que podem ocasionar o IAM que são: Diabetes Mellitus (DM), Hipertensão Arterial Sistêmica (HAS), Tabagismo, Obesidade, Sedentarismo, Estresse e consumo de álcool excessivo. Todos esses fatores facilitam o desenvolvimento das doenças cardiovasculares. No entanto, o conhecimento e o controle desses fatores são primordiais para a prevenção das doenças cardiovasculares, se adequando a prática de exercícios físicos, alimentação saudável, controle da HAS e do DM, não fumar, podendo minimizar o risco do IAM (PAIM; AZZOLIN; MORAES, 2012).

Um dos sintomas mais frequentes no IAM é a dor torácica, que é uma das causas de procura ao pronto-socorro, que tem um desafio clínico, devido à dificuldade da diferenciação dos diagnósticos não emergenciais e os altos riscos de morbimortalidade. Nessa avaliação de dor torácica aguda, existem dois tipos fundamentais para seu manejo: o exame físico onde se utiliza o eletrocardiograma (ECG) e marcadores de necrose miocárdica que são: Troponina, CK-MB entre 
outras e o manejo clínico, que se apresenta a angina, ou seja, uma queimação, pressão ou dificuldade para respirar, situada no precórdio ou em outra parte do tórax, com disseminação para o pescoço, ombro e braço esquerdo, tendo-se intensidade maior em poucos minutos, podendo ser acompanhada de sintomas como náuseas, vômitos e sudorese (RIBEIRO; YAMADA; BENVENUTI, 2014).

Desta forma, os profissionais da saúde devem ter um cuidado maior aos pacientes, fazendo um planejamento que seja real as suas necessidades, mantendo uma relação de confiança entre os envolvidos no cuidado, diálogo aberto, mostrando todas as informações necessárias sobre os riscos que levam ao IAM. Em relação aos fatores, devem ser modificados, como por exemplo, o tabagismo e o álcool, para que cessem o uso de tais substâncias fazendo com que haja o controle. Portanto, o estudo preconiza um melhor conhecimento sobre os principais fatores associados à incidência do IAM e também a prevenção aos hábitos de vida da população, diminuindo o alto índice de óbito associado a esta enfermidade.

O estudo tem como finalidade identificar os fatores de risco associados ao Infarto Agudo do Miocárdio (IAM).

\section{MÉTODO}

Trata-se de uma revisão integrativa da literatura, para a identificação de produções sobre os fatores de riscos associados ao IAM, entre 2011 a 2017 . Foi adotada a revisão integrativa da literatura, segundo Mendes e Galvão (2008), possibilitando, de forma sistemática e ordenada, o agrupamento de pesquisas científicas relevantes que serão analisadas e por final adquirir síntese de conclusão sobre o tema em estudo que possa da sua parte algumas decisões e melhorias da prática clínica.

A revisão integrativa da literatura sugere o estabelecimento de critérios bem precisos sobre a coleta de dados, análise e apresentação dos resultados. Dessa forma, foram adotadas as seis etapas constituintes da revisão integrativa da literatura: 1) seleção da pergunta de pesquisa; 2) definição dos critérios de inclusão 
e exclusão dos estudos e seleção da amostra; 3) representação dos estudos selecionados em formato de tabelas, considerando todas as características em comum; 4) análise criteriosa dos achados; 5) interpretação dos resultados e 6) apresentar a evidência encontrada (MENDES; SILVEIRA; GALVÃO, 2008).

Para conduzir a pesquisa, traremos como pergunta norteadora: Quais os fatores de riscos associados ao Infarto Agudo do Miocárdio (IAM)?

$\mathrm{Na}$ composição da produção científica e seleções de estudos na Biblioteca Virtual de Saúde (BVS) em português onde foram aplicados como bases de dados eletrônicos Scientific Eletronic Library onLine (SCIELO) que foram encontrados 2.383 artigos e Literatura Cientifica e Técnica da América Latina e Caribe (LILACS) onde foram descobertos 3.873 artigos, na qual as buscas dessas bases de dados ocorreram no período de fevereiro a dezembro de 2017. Os descritores utilizados foram: "Infarto" AND, "Sinais e Sintomas", "Fatores de Riscos", Os critérios de inclusão foram levados em consideração devido o alcance de todas as propostas literárias, indexação nas bases de dados referidas no período de 2011 a 2017. Foram selecionados como critério de exclusão artigos que antecederam o ano de 2011, que fugiram da temática proposta e artigos que apresentaram irrelevância com escassez de dados.

\section{RESULTADOS}

No que se refere à verificação bibliográfica, foram consultados as bases de dados identificando um total de 60 artigos onde fizemos uma exclusão dos artigos que fugiram do tema abordado, artigos que apresentaram ausência de informações, após estudos realizamos uma filtragem no qual foram selecionados 18 e utilizados 10. Esses dez artigos selecionados podem ser observados no quadro 1, 20\% (02) são do ano de 2012, 20\% (02) são do ano de 2014, 10\% (01) do ano de 2015, 40\% (04) são do ano de 2016 e 10\% (01) são do ano de 2017.

Em relação aos estudos abordados, foram observados as possíveis complicações que podem ser resultantes dos fatores de riscos associados ao IAM, 
aumentando os cuidados associados com relação aos fatores, HAS, DM, Sedentarismo, Obesidade, Consumo Excessivo de Álcool e Tabagismo. O quadro a seguir, mostra uma síntese de artigos incluídos na revisão integrativa apresentando o título, ano, autores, objetivos e resultados.

Quadro 1: Apresentação da síntese de artigos incluídos na revisão integrativa. Número/Título/Ano/Base de dados.

\begin{tabular}{|c|c|c|c|}
\hline $\mathbf{N}^{\circ}$ & Título & Ano & $\begin{array}{l}\text { Base de } \\
\text { dados }\end{array}$ \\
\hline 1 & $\begin{array}{l}\text { Prevalência de fatores de risco em pacientes com } \\
\text { infarto agudo do miocárdio. }\end{array}$ & 2016 & SCIELO \\
\hline 2 & $\begin{array}{l}\text { Associação entre adesão ao tratamento e tipos de } \\
\text { complicações cardiovasculares em pessoas com } \\
\text { Hipertensão Arterial. }\end{array}$ & 2016 & SCIELO \\
\hline 3 & $\begin{array}{l}\text { Associação de fatores de risco cardiovasculares com as } \\
\text { diferentes apresentações da síndrome coronariana } \\
\text { aguda. }\end{array}$ & 2014 & LILACS \\
\hline 4 & $\begin{array}{l}\text { Dor torácica no infarto agudo do miocárdio entre } \\
\text { pacientes diabéticos e não diabéticos. }\end{array}$ & 2012 & SCIELO \\
\hline 5 & $\begin{array}{l}\text { Controle Glicêmico Intensivo em Pacientes com Infarto } \\
\text { Agudo do Miocárdio: Tempo de Duração do Diabetes } \\
\text { Conta! }\end{array}$ & 2015 & SCIELO \\
\hline 6 & $\begin{array}{l}\text { Obesidade em Idosos e sua Associação com Consumo } \\
\text { Alimentar, Diabetes Mellitus e Infarto Agudo do } \\
\text { Miocárdio. }\end{array}$ & 2016 & LILACS \\
\hline 7 & $\begin{array}{l}\text { Associação Entre a Obesidade Central e a Incidência de } \\
\text { Doenças e Fatores de Risco Cardiovascular. }\end{array}$ & 2017 & LILACS \\
\hline 8 & $\begin{array}{l}\text { Fatores de risco que podem levar ao Infarto Agudo do } \\
\text { Miocárdio (IAM), e o papel do Enfermeiro na sua } \\
\text { prevenção. }\end{array}$ & 2012 & LILACS \\
\hline 9 & $\begin{array}{l}\text { Abuso de álcool após Síndrome Coronariana Aguda: } \\
\text { avaliação prospectiva no estudo ERICO. }\end{array}$ & 2014 & SCIELO \\
\hline 10 & $\begin{array}{lll}\text { Sofrimento psicológico relacionado à cessação do } & \text { do } \\
\text { tabagismo em pacientes com infarto agudo do } \\
\text { miocárdio. }\end{array}$ & 2016 & SCIELO \\
\hline
\end{tabular}

No segundo quadro abaixo, com base na análise dos artigos utilizados na revisão integrativa, foi fundamental questionar os fatores de riscos associados ao IAM, como é o caso do Sedentarismo, Obesidades, HAS, DM, Consumo de álcool excessivo e Tabagismo. 
Quadro 2: Apresentação da síntese de artigos incluídos na revisão integrativa. Autores/Objetivos/Resultados.

\begin{tabular}{|c|c|c|c|}
\hline $\mathrm{N}^{\circ}$ & Autores & Objetivos & Resultados \\
\hline 1 & $\begin{array}{l}\text { MERTINS } \\
\text { et al., }\end{array}$ & $\begin{array}{l}\text { Identificar a prevalência de } \\
\text { fatores de risco em pacientes } \\
\text { com infarto agudo do } \\
\text { miocárdio, internados em } \\
\text { uma Unidade de Terapia } \\
\text { Intensiva Coronariana de um } \\
\text { hospital da região noroeste } \\
\text { do estado do Rio Grande do } \\
\text { Sul Brasil. }\end{array}$ & $\begin{array}{l}\text { A prevalência dos fatores de } \\
\text { risco se distribuiu da seguinte } \\
\text { forma: sedentarismo }(91,7 \%) \text {, } \\
\text { hipertensão arterial sistêmica } \\
(63,8 \%) \text {, estresse }(50 \%) \text {, } \\
\text { circunferência abdominal } \\
\text { alterada }(50 \%) \text {, história familiar } \\
(43,7 \%) \text {, tabagismo }(41,7 \%) \text {, } \\
\text { sobrepeso (35,5\%), obesidade } \\
(33,4 \%) \text { dislipidemia }(23 \%) \text {, } \\
\text { diabetes mellitus }(20,8 \%) \text { e } \\
\text { consumo de álcool }(12,5 \%) .\end{array}$ \\
\hline 2 & LIMA et al., & $\begin{array}{l}\text { Verificar a associação entre } \\
\text { adesão ao tratamento anti- } \\
\text { hipertensivo e tipos de } \\
\text { complicações } \\
\text { cardiovasculares } \\
\text { em presentes } \\
\text { Hipertensão } \\
\text { Sistêmica. }\end{array}$ & $\begin{array}{l}\text { As complicações mais } \\
\text { prevalentes entre a adesão e } \\
\text { tratamento anti-hipertensiva } \\
\text { foram: acidente vascular } \\
\text { cefálico }(37,9 \%) \text {, seguido de } \\
\text { infarto agudo do miocárdio } \\
(20,3 \%) \text { Os resultados } \\
\text { demonstram a necessidade de } \\
\text { mudança comportamental da } \\
\text { clientela hipertensa com } \\
\text { adoção de medidas de } \\
\text { promoção da saúde a fim de } \\
\text { prevenir novos agravos } \\
\text { cardiovasculares. }\end{array}$ \\
\hline 3 & $\begin{array}{l}\text { BRUNORI } \\
\text { et al., }\end{array}$ & $\begin{array}{l}\text { Identificar a relação das } \\
\text { diferentes apresentações da } \\
\text { Síndromer Coronariana } \\
\text { Aguda (SCA) com fatores de } \\
\text { risco cardiovasculares entre } \\
\text { indivíduos hospitalizados. }\end{array}$ & $\begin{array}{l}\text { Com relação à SCA envolvendo } \\
\text { fatores de riscos } \\
\text { cardiovasculares observou-se } \\
\text { que houve predomínio de } \\
\text { infarto agudo do miocárdio com } \\
\text { supradesnivelamento Houve } \\
\text { segmento ST. dignificativa de } \\
\text { associação do } \\
\text { hipertensão arterial sistêmica } \\
\text { com angina instável e altos } \\
\text { valores de lipoproteína de baixa } \\
\text { densidade com infarto, sem } \\
\text { influência de características } \\
\text { sociodemográficas. }\end{array}$ \\
\hline
\end{tabular}




\begin{tabular}{|c|c|c|c|}
\hline 4 & $\begin{array}{l}\text { PAIM, } \\
\text { AZZOLIN, } \\
\text { MORAES }\end{array}$ & $\begin{array}{l}\text { Comparar a presença e } \\
\text { intensidade de dor torácica } \\
\text { no IAM entre pacientes } \\
\text { diabéticos e não diabéticos. }\end{array}$ & $\begin{array}{l}\text { O estudo mostrou semelhança } \\
\text { na presença e intensidade da } \\
\text { dor torácica entre pacientes } \\
\text { diabéticos e não diabéticos } \\
\text { acometidos de IAM. }\end{array}$ \\
\hline 5 & $\begin{array}{l}\text { FERREIRA } \\
\text { et al., }\end{array}$ & \begin{tabular}{llr} 
Avaliar & o & \multicolumn{2}{r}{ impacto } \\
prognóstico & do & tempo de \\
duração & e & controle \\
metabólico do DMTII em uma \\
população hospitalizada por \\
SCA.
\end{tabular} & 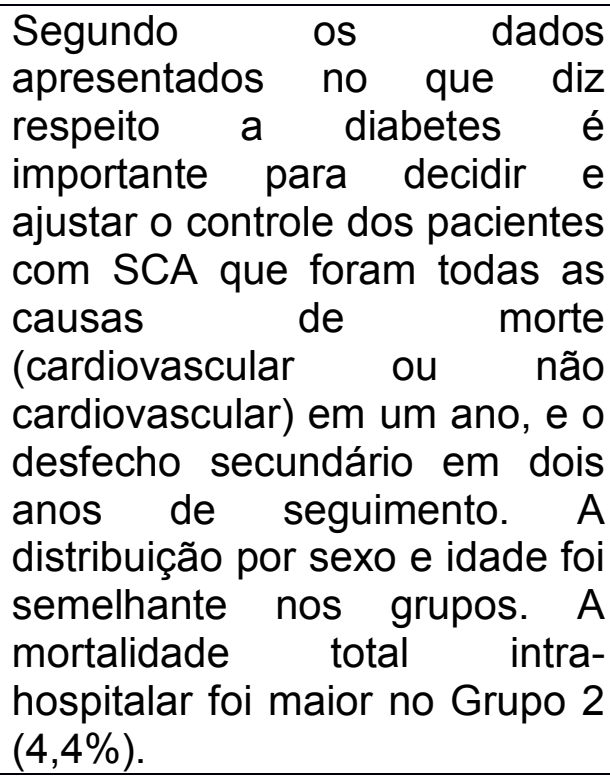 \\
\hline 6 & $\begin{array}{l}\text { SILVEIRA } \\
\text { et al., }\end{array}$ & $\begin{array}{l}\text { Investigar a prevalência de } \\
\text { obesidade e fatores } \\
\text { associados, com ênfase na } \\
\text { presença de outras doenças } \\
\text { e no consumo alimentar, em } \\
\text { idosos usuários do Sistema } \\
\text { Único de Saúde (SUS). }\end{array}$ & $\begin{array}{l}\text { A obesidade teve elevada } \\
\text { prevalência na população idosa } \\
\text { estudada e esteve associada a } \\
\text { outras doenças, nos quais os } \\
\text { fatores associados à obesidade } \\
\text { foram idade de } 60-69 \text { e } 70-79 \\
\text { anos, consumo inadequado de } \\
\text { cereais integrais e frutas, } \\
\text { doenças osteomusculares, } \\
\text { diabetes mellitus e infarto } \\
\text { agudo do miocárdio. }\end{array}$ \\
\hline 7 & $\begin{array}{l}\text { BARROSO } \\
\text { et al., }\end{array}$ & $\begin{array}{lcr}\text { Avaliar a associação da } & \text { da } \\
\text { obesidade central com a } & \text { a doenças e } \\
\text { incidência de do disco } \\
\text { fatores de } \\
\text { cardiovascular. }\end{array}$ & $\begin{array}{l}\text { A obesidade central esteve } \\
\text { associada a uma maior } \\
\text { incidência de desenvolvimento } \\
\text { de fatores de risco relacionados } \\
\text { a doenças cardiovasculares } \\
\text { onde as Mulheres }(\mathrm{n}=39) \text {, com } \\
\text { idade média de } 44,18 \pm 14,42 \\
\text { anos, sendo } 70 \% \text { obesos e } \\
38 \% \text { hipertensos } \\
\text { corresponderam à maioria da } \\
\text { amostra estudada. }\end{array}$ \\
\hline 8 & CHAGAS & $\begin{array}{l}\text { Mostrar que as doenças } \\
\text { cardiovasculares } \\
\text { principalmente o IAM, estão } \\
\text { diretamente ligados a fatores }\end{array}$ & \begin{tabular}{lrrr} 
Este estudo & \multicolumn{2}{c}{ mostra } & as \\
consequências de & se adotar \\
este novo estilo de & vida \\
sedentário, & as & & suas \\
\end{tabular} \\
\hline
\end{tabular}




\begin{tabular}{|c|c|c|c|}
\hline & & $\begin{array}{l}\text { de riscos, dessa forma } \\
\text { mudanças nos hábitos de } \\
\text { vida e na prevenção primária } \\
\text { destes fatores podem levar a } \\
\text { população a uma diminuição } \\
\text { de doenças } \\
\text { cardiovasculares. }\end{array}$ & $\begin{array}{l}\text { complicações futuras que estão } \\
\text { diretamente ligadas aos fatores } \\
\text { de riscos das doenças arterias } \\
\text { crônicas (DAC) e o infarto } \\
\text { agudo do miocárdio (IAM). Que } \\
\text { com práticas de saúde } \\
\text { saudáveis podemos evitar } \\
\text { doenças arteriais, e os preparar } \\
\text { para uma vida futura mais } \\
\text { saudável longe de doenças } \\
\text { cardiovasculares e das } \\
\text { estatísticas que hoje são tão } \\
\text { exorbitantes. }\end{array}$ \\
\hline 9 & $\begin{array}{l}\text { MORILHA } \\
\text { et al., }\end{array}$ & $\begin{array}{l}\text { Investigar a relação entre } \\
\text { abuso de álcool após } \\
\text { Síndrome Coronariana } \\
\text { Aguda (SCA) na estratégia } \\
\text { de registros de Insuficiência } \\
\text { Coronariana (ERICO). }\end{array}$ & $\begin{array}{l}\text { As frequências de abuso de } \\
\text { álcool foram } 24,7 \% \text { e } 21,1 \% \\
\text { nos } 12 \text { meses anteriores à SCA } \\
\text { e seis meses após, } \\
\text { respectivamente. Os fatores de } \\
\text { risco cardiovascular mais } \\
\text { significativos associados com } \\
\text { alto risco de abuso de álcool } 30 \\
\text { dias após o evento agudo } \\
\text { foram: sexo masculino (88,9\%), } \\
\text { tabagismo atual (52,8\%) e } \\
\text { hipertensão }(58,3 \%) \text {. }\end{array}$ \\
\hline 10 & $\begin{array}{l}\text { SANTOS, } \\
\text { GODOY, } \\
\text { GODOY }\end{array}$ & \begin{tabular}{llr} 
Analisar as evidências & \multicolumn{2}{c}{ as } \\
científicas & \multicolumn{2}{c}{ acerca do do } \\
sofrimento psicológico na \\
cessação do tabagismo \\
envolvendo pacientes que \\
tiveram infarto agudo do \\
miocárdio.
\end{tabular} & $\begin{array}{l}\text { Este estudo de revisão } \\
\text { aumenta o conhecimento do } \\
\text { tema e mostra a necessidade } \\
\text { de se investir em pesquisas } \\
\text { futuras que analisam subgrupos } \\
\text { de fumantes com as principais } \\
\text { comorbidades relacionadas ao } \\
\text { uso do tabaco, como o infarto } \\
\text { agudo do miocárdio. }\end{array}$ \\
\hline
\end{tabular}

\section{DISCUSSÃo}

As doenças cardiovasculares apresentam a principal causa de mortalidade em todo o mundo, em relação aos fatores de risco para doenças cardíacas, foi observado que o estresse tem uma prevalência de $50 \%$ para o desenvolvimento do IAM. Por isso é de suma importante ter o conhecimento e fazer o acompanhamento 
desses fatores para se ter o controle, diminuindo a doença isquêmica (MERTINS et al., 2016).

Pode-se perceber na literatura que Mertins et al., (2016) e Jordão et al., (2016) afirmam que o estresse é classificado um dos fatores principais por várias alterações do estado de saúde da população mundial, ocasionando alterações fisiológicas, comportamentais, cognitivas, podendo levar ao surgimento de doenças ou até a morte, o estresse apresenta um processo psicofisiológico do organismo que é essencial para diagnosticar respostas desencadeadas da forma como os estímulos são processados.

Segundo Lima et al. (2016), a HAS é apresentada em todo o mundo como fator de risco importante para as doenças cardiovasculares, sabe-se que a HAS é um problema de saúde cada vez mais comum em relação a sua evolução que em muitos casos se torna assintomática e lenta. Na maioria das vezes, ela só se torna perceptível apenas, quando acontece um evento cardiovascular, onde a condição de vida poderá ser comprometida ou até ocasionar a morte. Os estudos mostram que pessoas com problemas de hipertensão só começam a se submeter ao tratamento quando aparecem as complicações, como: IAM, doença arterial coronariana (DAC), insuficiência cardíaca congestiva (ICC) entre outras, quando se apresenta esses problemas cardiovasculares tem-se uma aceitação ao tratamento para o monitoramento da pressão arterial, fazendo assim o controle do aparelho cardiovascular.

Correlacionando o pensamento Lima et al., (2016) e Brunori et al., (2014) evidencia que as mortes por IAM apresentam $12,1 \%$, dentre os fatores associados ao desenvolvimento de doenças cardiovasculares existem estudos onde se tem investigações em relação a todos os riscos cardiovasculares, tal informação serve para o aumento do conhecimento e fortalecimento dos profissionais da saúde em relação aos fatores. A Sociedade Brasileira de Cardiologia aborda o controle das principais causas como, HAS, DM, entre outros, contudo destacando-os como marcadores de pior prognóstico entre pessoas com IAM.

O DM apresenta a quinta causa de morte no mundo, com relação as suas complicações, consta que as doenças cardiovasculares decorrem com maior frequência, tornando-se também responsáveis por uma elevada mortalidade. Sendo 
que, as comorbidades relacionadas ao DM aumentam o risco cardiovascular exigindo cuidados maiores para a prevenção do IAM (PAIM; AZZOLIN; MORAES, 2012).

Fazendo-se a comparação sobre diabetes, Ferreira et al., (2015), nos mostra que os pacientes que apresentam IAM, precocemente diagnosticados com DM, desenvolve um pior prognóstico ao menos prazo do que os não diabéticos. Apesar de várias evoluções no tratamento e diagnóstico da doença cardiovascular, exclusivamente no tratamento da síndrome coronariana aguda, com um grande impacto sobre a mortalidade e morbidade do IAM.

A obesidade é uma séria complicação para a saúde pública, o domínio da obesidade em outro maior estudo populacional não passou de $35 \%$, vários estudos descobriram essa associação, que pode ser ocasionado a sobrevivência, ou seja, uma diminuída expectativa de vida em relação as pessoas obesas, que dificilmente chegam aos 80 anos de idade. Em relação a obesidade e o IAM é especificada, entretanto, com particularidades nas pessoas idosas, a taxa de mortalidade a respeito de outras doenças são relacionadas a obesidade e sobrepeso, chamandose de paradoxo da obesidade, mas os estudos mostraram com clareza que o risco da obesidade pode gerar o IAM (SILVEIRA et al., 2016).

Segundo Barroso et al., (2017), a obesidade é estabelecida como excesso de gordura que resulta no desequilíbrio crônico por meio do consumo alimentar e gasto energético que vem aumentando e adquirindo proporções alarmantes. O consumo exagerado de alimentos gordurosos e açucares estão relacionados ao aumento da obesidade e ao desenvolvimento de doenças cardiovasculares.

Silveira et al., (2016); Barroso et al., (2017) e Coelho et al., (2011), corroboram com a mesma teoria onde a obesidade é um fator para o desenvolvimento de doenças do aparelho cardiovascular, a obesidade pode ser definida como um padrão de massa corporal, tendo um crescimento de forma epidêmica na sociedade, favorecendo assim, o surgimento das doenças cardiovasculares por estar dentro de processos metabólicos associados a diversos fatores de riscos. Entretanto, muitos estudos têm apontado um "paradoxo da obesidade", onde a obesidade parece ser protetora contra um parece adverso, marcando uma diminuição da mortalidade intra-hospitalar e a um grande prazo. 
Os fatores de risco são condições que levam uma pessoa a um risco maior de desenvolver doenças cardíacas, existem vários fatores para o desenvolvimento de doenças cardiovasculares, tendo uma ênfase maior no sedentarismo. Onde a falta de atividade física é um fator importante para gerar doenças coronárias, quando se tem a prática de exercícios físicos regulares, vigorosos e moderados tem-se um papel essencial na prevenção de doenças do aparelho cardiovascular (CHAGAS, 2012).

Gualano; Tinucci, (2011) diz que concorda com Chagas et al., (2012) quando diz que a falta da prática de atividade física aumenta consideravelmente a incidência relativa de doença arterial coronariana, IAM, HAS, Câncer de Cólon, Câncer de Mama, Osteoporose e DM. A falta de atividade física é um dos maiores problemas de saúde pública, entretanto é considerado que, $70 \%$ da população não chegamos ao mínimo de atividade física estabelecida, onde muitas doenças podem ser evitadas através de exercícios físicos possivelmente evitando vários tipos de doenças.

Já para Pereira et al., (2017), o estilo de vida é um conjunto de práticas cotidianas que retrata os valores e atitudes das pessoas, contudo, esses hábitos e ações responsáveis que se associam a qualidade de vida percebida e intrínseca ao indivíduo. O princípio de estilo de vida mostra que isso vai bem além da prática ou não de exercício físico, onde aquele que tem uma vida ativa demonstra benefícios totalmente ligados à saúde. Conhecendo a realização na prática de exercícios, acontece uma regulação no estilo de vida mais saudável, se tornando de extrema relevância para a saúde pública.

O fator principal de risco para doenças em países com desenvolvimento é o álcool e também o terceiro fator nos países desenvolvidos, é interessante abordar que os padrões de consumo do álcool não foram relacionados só em desfechos agudos de saúde, como os danos físicos, mas também, a patologias crônicas e umas das principais são as doenças cardiovasculares (MORILHA et al., 2014).

Segundo Rozin; Zagonel (2012), relaciona seu pensamento ao de Morilha et al., (2014) quando diz que a relação ao álcool também deve ser levado em consideração por ser uma droga psicoativa, de mais utilidades em vários países, quando se tem o uso precoce do álcool se obtém um risco maior para o 
desenvolvimento de dependência e doenças graves como fatores já citados que podem ocasionar o IAM. O álcool é o tipo de droga psicoativa mais utilizada na maioria dos países, os seus danos a saúde são muito altos, depois do tabaco é a segunda causa de mortes decorrentes de várias doenças e de uso de drogas.

A exposição ao tabaco é um dos riscos principais de bastantes comobirdades, que leva a um evento de doenças que podem ser fatais, especialmente as doenças cardiovasculares, aterosclerótica, entre outras, os fumantes vivem mais ou menos dez anos a menos do que os que não fumam, tendo duas vezes mais o risco de ocorrer eventos cardíacos em média de dez anos, sendo assim, o percentual para o IAM é elevado. A nicotina é considerada como droga por gerar dependência e por ser lícita, portanto o tabagismo é um problema na saúde pública mundial, encarregado do maior número de mortes no mundo, onde seus efeitos são negativos e acarretam complicações econômicas desfavoráveis, com relação a sua mortalidade o tabaco mata uma pessoa a cada seis segundos sendo responsável a cada dez mortes em adultos (SANTOS; GODOY; GODOY, 2016).

Já Nunes et al., (2011), aborda que o controle do tabaco tem uma prioridade na saúde pública, uma vez que, as causas de mortes podem ser prevenidas, e o tabagismo é a maior delas, esse é um tempo muito abrangente, no qual o controle para ser bem sucedido necessita de uma implantação em seus diversos componentes: exposição tabágica e cessação, produção, prevenção e políticas de saúde.

\section{CONCLUSÃO}

O IAM é uma das doenças cardiovasculares mais graves, decorrente da oclusão do vaso, dificultando a passagem do fluxo sanguíneo para o coração, causando a morte das células cardíacas. As doenças cardiovasculares são as maiores em morte no mundo, entre elas o principal é o IAM.

Este estudo mostra os principais riscos que desencadeiam o IAM evidenciando o grau elevado de morbimortalidade, mostrando que a população 
precisa ter a consciência de hábitos saudáveis, tendo também o conhecimento sobre todos os fatores para que haja uma prevenção precoce, evitando assim, essa enfermidade. Nesse contexto, também é importante ressaltar que todos os profissionais da área da saúde tenham o conhecimento de todos os fatores de risco como DM, HAS, Sedentarismo, Obesidade, Consumo Excessivo de Álcool e Tabagismo, expondo que quando não se há o controle dos fatores podem acarretar o IAM, e na maioria das vezes levar a morte.

\section{REFERÊNCIAS BIBLIOGRÁFICAS}

BRUNORI, Evelise Helena Fadini Reis et al. Associação de fatores de risco cardiovasculares com as diferentes apresentações da síndrome coronariana aguda. Revista Latino-Americana de Enfermagem, v. 22, n. 4, p. 538-546, 2014.

BARROSO, Taianah Almeida et al. Associação Entre a Obesidade Central e a Incidência de Doenças e Fatores de Risco Cardiovascular. International Journal of Cardiovascular Sciences, v. 30, n. 5, p. 416-424, 2017.

CHAGAS, J. P. S., Fatores de risco que podem levar ao infarto agudo do miocárdio (IAM), e o papel do Enfermeiro na sua prevenção, Fundação Educacional do Município de Assis FEMA - Assis, 27p. 2012.

COELHO, dos L. S., CANTARELLI, M. J. de C., JÚNIOR, H. J. C., Impacto da obesidade nos resultados hospitalares da intervenção coronária percutânea: resultados do registro do hospital bandeirantes. Revista Brasileira de Cardiologia Invasiva.: vol.19, №4 ISSN 0104-143.2011.

FERREIRA, Ana Rita Pereira Alves et al. Controle Glicêmico Intensivo em Pacientes com Infarto Agudo do Miocárdio: Tempo de Duração do Diabetes Conta! Int J Cardiovasc Sci, v. 28, n. 6, p. 487-495, 2015.

GUALANO, Bruno; TINUCCI, Taís. Sedentarismo, exercício físico e doenças crônicas. Revista Brasileira de Educação Física e Esporte, v. 25, n. spe, p. 37-43, 2011.

HUGUENIN, Felipe Machado et al. Caracterização dos padrões de variação dos cuidados de saúde a partir dos gastos com internações por infarto agudo do miocárdio no Sistema Único de Saúde. Revista Brasileira de Epidemiologia, v. 19, p. 229-242, 2016.

VASCONCELLOS DE LIMA COSTA, Eduardo Tavares; TRAJANO, Lima. Possíveis fatores estressantes na unidade de terapia intensiva neonatal em hospital universitário. Ver Bras Ter Intensiva, v. 28, n. 3, p. 310-314, 2016.

BRAZ DA SILVA LIMA, Daniele et al. Associação entre adesão ao tratamento e tipos de complicações cardiovasculares em pessoas com hipertensão arterial. Texto \& Contexto Enfermagem, v. 25, n. 3, 2016.

MATHIONI MERTINS, Simone et al. Prevalência de fatores de risco em pacientes com infarto agudo do miocárdio. Avances em Enfermería, v. 34, n. 1, p. 30-38, 2016.

MENDES, Karina Dal Sasso et al. Revisão integrativa: método de pesquisa para a incorporação de evidências na saúde e na enfermagem. Texto \& Contexto-Enfermagem, v. 17, n. 4, p. 758- 
$764,2008$.

MORILHA, Abner et al. Abuso de álcool após síndrome coronariana aguda: avaliação prospectiva no estudo ERICO. 2015.

NUNES, Sandra Odebrecht Vargas; CASTRO, Márcia Regina Pizzo de. Tabagismo: abordagem, prevenção e tratamento. 2010.

PAIM, Crislaine Pires Padilha; AZZOLIN, Karina de Oliveira; MORAES, Maria Antonieta Pereira de. Dor torácica no infarto agudo do miocárdio entre pacientes diabéticos e não diabéticos. Revista brasileira de enfermagem. Vol. 65, n. 1 (jan./fev. 2012), p. 77-82, 2012.

DE LOURENÇO PEREIRA, Daniela Santos; DE OLIVEIRA, Anamaria Siriani; DE OLIVEIRA, Rinaldo Eduardo Machado. AVALIAÇÃO DO PERFIL DE ESTILO DE VIDA DE USUÁRIOS QUE PRATICAM EXERCÍCIO FÍSICO REGULAR COMPARADO A USUÁRIOS SEDENTÁRIOS INSERIDOS EM UMA ESTRATÉGIA DE SAÚDE DA FAMÍLIA-REGIÃO SUDESTE DO BRASIL. Revista de APS, v. 20, n. 1, 2017.

RIBEIRO, W. N., YAMADA, A. T., BENVENUTI, L. A., Homem de 66 anos com infarto agudo do miocárdio e morte em assistolia após angioplastia coronária primária. Arquivo Brasileiro Cardiologia; 103(3): de 31-e 36.2014.

ROZIN, Leandro; ZAGONEL, Ivete Palmira Sanson. Fatores de risco para dependência de álcool em adolescentes. 2012.

MOREIRA-SANTOS, Thyego Mychell; GODOY, Irma; DE GODOY, Ilda. Sofrimento psicológico relacionado à cessação do tabagismo em pacientes com infarto agudo do miocárdio. Jornal Brasileiro de Pneumologia, v. 42, n. 1, p. 61-67, 2016.

SILVEIRA, E. A., VIEIRA, L. L., JARDIM, T. V., SOUZA, J. D., Obesidade em idosos e sua associação com consumo alimentar, diabetes mellitus e infarto agudo do miocárdio. Arquivo Brasileiro Cardiologia; v. 107(6): p. 509-517. 2016. 